\author{
Marquette University \\ e-Publications@Marquette
}

6-15-2004

\title{
Characterization of Epoxy Resin (SU-8) Film Using Thickness- Shear Mode (TSM) Resonator under Various Conditions
}

Jeanne Hossenlopp

Marquette University, jeanne.hossenlopp@marquette.edu

Lizhong Jiang

Marquette University

Richard Cernosek

Marquette University

Fabien Josse

Marquette University, fabien.josse@marquette.edu

Follow this and additional works at: https://epublications.marquette.edu/chem_fac

Part of the Chemistry Commons

\section{Recommended Citation}

Hossenlopp, Jeanne; Jiang, Lizhong; Cernosek, Richard; and Josse, Fabien, "Characterization of Epoxy Resin (SU-8) Film Using Thickness-Shear Mode (TSM) Resonator under Various Conditions" (2004).

Chemistry Faculty Research and Publications. 6.

https://epublications.marquette.edu/chem_fac/6 


\title{
Characterization of Epoxy Resin (SU-8) Film Using Thickness-Shear Mode (TSM) Resonator Under Various Conditions
}

\author{
By Jeanne Hossenlopp, Lizhong Jiang, Richard Cernosek, Fabien Josse
}

Characterization of an epoxy resin film, commonly known as SU-8, is presented using thickness shear mode (TSM) quartz resonator. The impedance-admittance characteristics of the equivalent circuit models of the unperturbed and coated resonators are analyzed to extract the storage modulus and loss modulus ( $G^{\prime}$ and $\left.G^{\prime \prime}\right)$. Those parameters are needed to establish SU-8 film as an effective wave-guiding layer in the implementation of guided shear-horizontal surface acoustic wave (SH-SAW) sensor platforms. Both cured and uncured polymer films are studied at the fundamental and third harmonic frequencies of the TSM resonators. The storage modulus $\left(G^{\prime}\right)$ and loss modulus $\left(G^{\prime \prime}\right)$ of the SU-8 film approach constant values of $1.66 \times 10^{10}$ dyne $/ \mathrm{cm}^{2}$ and $6.0 \times 10^{8} \mathrm{dyne} / \mathrm{cm}^{2}$, respectively, for relatively thicker films (>20 $\left.\mu \mathrm{m}\right)$ at a relatively low frequency of $9 \mathrm{MHz}$. The most accurate values for the extracted shear moduli $G$ $\left(G=G^{\prime}+j G^{\prime \prime}\right)$ are obtained at high thickness where the viscoelastic contribution to the TSM response is substantial. The effect of temperature on the storage and loss moduli is determined for the range of -75 to $40 \mathrm{C}$. It is found that the polymer approaches a totally glassy state below $-60 \mathrm{C}$. Exposure to water appears to follow Fickian diff usion behavior at short times and this exposure also results in changes to both $G^{\prime}$ and $G^{\prime \prime}$. However, stability is rapidly reached with exposure to water, indicating relatively lower water absorption, consistent with the extracted diffusion coefficient.

\section{Introduction}

Thin polymer films are commonly used in the implementation of chemical and biochemical sensors, as partially selective and sensitive coatings for the detection of chemical agents, ${ }^{1-4}$ or as wave-guiding layers in guided shear-horizontal surface acoustic wave (SH-SAW) sensor platforms. ${ }^{5}$ The design and implementation of these sensors rely on the characteristic information about the viscoelastic properties of the polymer films and subsequent changes upon exposure to environmental factors, such as liquid contact and temperature change. Several technologies and techniques can be employed in characterizing the thin viscoelastic films. However, the use of the thickness-shear mode (TSM) quartz resonator is proposed here for rapid characterization because of its simplicity and ability to support shear horizontal waves, which are ideal for the characterization of the complex shear modulus of thin viscoelastic films. 
The TSM resonator is very sensitive to surface perturbations. The surface perturbations include surface mechanical loadings such as an added ideal mass layer, a viscoelastic layer, and/or a contacting liquid. Two methods have been used to interpret TSM resonator response depending on the properties of perturbation: the Sauerbrey equation, and the crystal impedance method. The Sauerbrey equation relates mass accumulated on the crystal to the change in the resonant frequency. ${ }^{6}$ However, this method is only valid for a rigid and acoustically thin film. Because the mass effect is not the only effect that influences the acoustic behavior of the TSM device, the Sauerbrey method is unable to distinguish between changes in mass load on the surface and the viscosity changes of the liquid or changes in the viscoelastic properties of the coating. To overcome these limitations, the crystal impedance method has been developed. ${ }^{7-9}$ This method allows the extraction of physical parameters (viscosity, density, shear modulus) from loading on the TSM resonator.

The TSM resonator consists of a thin disk of AT-cut quartz with metal electrodes deposited on both sides. Due to the piezoelectric properties and crystalline orientation of the quartz, application of alternating potential to the electrodes of the resonator results in an internal mechanical stress, and consequently, a shear deformation of the crystal. Resonance will occur when the crystal thickness is an odd multiple of half the acoustic wavelength. If a medium is in contact with one or both sides of the resonator surfaces, the excitation of the crystal subjects the medium on the crystal to an oscillatory driving force. Due to the electromechanical coupling, the mechanical properties of the contacting medium are reflected in the electrical response of the resonator.

The crystal impedance method involves using a network analyzer to measure the reflection coefficient, $S_{11}$, of the surface-loaded TSM quartz resonator. The admittance can be obtained, in the form of complex quantities, from the reflection coefficient. It is convenient to use an equivalent circuit model to describe the electrical behavior of the TSM resonator. With only a few lumped elements, the modified Butterworth-VanDyke (BVD) model simulates the electrical characteristics of the TSM resonator over a range of frequencies near resonance. This model can explicitly relate the lumped elements in the circuit to physical properties of the TSM, as well as the surface loading (liquid load and/or viscoelastic film). Fitting the circuit model to the measured admittance data allows the extraction the physical properties of the surface perturbation. $^{10}$

SU-8 is an epoxy-based negative photoresist, a glycidyl ether derivative of bisphenol A novolac. ${ }^{11}$ Due to the high level of epoxy group functionality, which allows for high crosslink density, SU-8 films are thermally and chemically stable, and thus offer the possibility of use as 
stable wave-guiding layers on SH-SAW sensor platforms. To explore the suitability of SU-8 for this application, characterization of film viscoelastic properties and determination of the rate of water uptake are necessary. In the present article, use of a TSM resonator for relatively rapid extraction of the viscoelastic properties of SU-8 films, and extraction of the water diffusion coefficient, is presented.

\section{Theory}

The BVD model can be used to describe both the unperturbed and perturbed quartz crystals as shown in Figure 1. Figure 1(a) represents the unperturbed TSM resonator, which consists of two branches. A static capacitance $C_{0}$ arises between the electrodes located on opposite sides of the insulating quartz. A parasitic capacitance $C_{\mathrm{p}}$ also arises due to the test fixture. The net capacitance of the static branch, $C_{0}{ }^{*}$, is the sum of $C_{0}$ and $C_{\mathrm{p}}$. Because the quartz is also piezoelectric, electromechanical coupling gives rise to additional motional contributions $\left(L_{1}, C_{1}\right.$, and $\left.R_{1}\right)$. The static branch dominates the electrical behaviors away from resonance, while the motional branch dominates near resonance. The modified BVD model shown in Figure 1(b) can be used to describe the electrical response of the perturbed device. When the resonator has a surface perturbation, the motional impedance increases, as indicated by the introduction of the complex electrical load impedance $Z_{\mathrm{e}}$ in Figure 1 (b). ${ }^{8}$

$$
Z_{e}=\frac{N \pi}{4 K^{2} \omega_{\mathrm{s}} C_{0}}\left(\frac{Z_{\mathrm{s}}}{Z_{\mathrm{q}}}\right)
$$

where $N$ is the vibration mode index, $K^{2}$ is the square of the quartz electromechanical coupling coefficient, $\omega_{\mathrm{s}}$ is the angular resonant frequency for the unperturbed TSM resonator, $Z_{\mathrm{q}}=$ $\left(\rho_{\mathrm{q}} \mu_{\mathrm{q}}\right)^{1 / 2}$ is the quartz shear wave characteristic impedance, where $\rho_{\mathrm{q}}$ and $\mu_{\mathrm{q}}$ are the mass density and shear stiffness of the quartz, and $Z_{\mathrm{s}}$ is the shear mechanical impedance at the device surface: ${ }^{12}$

$$
Z_{\mathrm{s}}=\left.\frac{T_{\mathrm{xy}}}{v_{\mathrm{x}}}\right|_{\mathrm{y}=0}
$$

where $T_{\mathrm{xy}}$ is the peak sinusoidal steady state shear stress imposed on the contacting medium by the resonator and $v_{\mathrm{x}}$ is the resulting $x$-directed surface shear particle velocity displacement. It is noted tha $Z_{\mathrm{s}}$ is a complex quantity.

Letting $Z_{\mathrm{e}}=R_{2}+j \omega L_{2}$, the electrical impedance element $R_{2}$ and $L_{2}$ can be related to the real and imaginary components of the surface mechanical impedance as: 


$$
\begin{aligned}
& R_{2}=\frac{N \pi}{4 K^{2} \omega_{\mathrm{s}}^{2} C_{0}}\left(\frac{\operatorname{Re}\left(Z_{\mathrm{s}}\right)}{Z_{\mathrm{q}}}\right) \\
& L_{2}=\frac{N \pi}{4 K^{2} \omega_{\mathrm{s}} C_{0}}\left(\frac{\operatorname{Im}\left(Z_{\mathrm{s}}\right)}{Z_{\mathrm{q}}}\right)
\end{aligned}
$$

A general model has been developed ${ }^{9}$ that can incorporate a physically diverse set of single-component loads, including rigid solids, viscoelastic media, and fluids. In general, multiple surface loadings on the TSM resonator cannot be treated in a linear fashion except for ideal mass layer. In the case of a TSM resonator with simultaneous viscoelastic and liquid loading, the total impedance is not equal to the sum of the characteristic impedance of the individual layers. This is due to the phase shift caused by the viscoelastic layer. Instead, the total surface mechanical impedance at the viscoelastic coating/liquid interface can be defined as:12,13

$$
Z_{\mathrm{s}}=Z_{0}\left[\frac{Z_{1} \cosh \left(\beta h_{f}\right)+Z_{0} \sinh \left(\beta h_{f}\right)}{Z_{0} \cosh \left(\beta h_{f}\right)+Z_{1} \sinh \left(\beta h_{f}\right)}\right]
$$

where, $Z_{0}$ and $Z_{1}$ are the characteristic mechanical impedance of the viscoelastic film and the Newtonian fluid, respectively. $\beta=j \omega\left(\rho_{f} / G\right)^{1 / 2}$ is the complex wave propagation constant. $\rho_{f}$ and $h_{f}$ are the density and thickness of the film, respectively. The parameters for AT-cut quartz are readily available from the literature. The remaining parameters are measured experimentally or estimated based on available data in the literature ${ }^{.12}$ In eq (4), the complex wave propagation constant, $\beta$, is equal to $j \omega\left(\rho_{f} / G\right)^{1 / 2}$ and $Z_{1}$ is the characteristic mechanical impedance of the liquid layer in contact with the coating. Without loss of generality, eq (4) can be rearranged into a more useful form:

$$
Z_{\mathrm{S}}=\frac{Z_{1}+Z_{0} \tanh \left(\beta h_{f}\right)}{1+\left(Z_{1} / Z_{0}\right) \tanh \left(\beta h_{f}\right)}
$$

The combined surface effects due to the viscoelastic coating and the liquid overlayer are heavily dependent on the impedance ratio, $Z_{1} / Z_{0}$, at the coating/liquid interface. If $Z_{1}$ is comparable in magnitude to $Z_{0}$, the shear acoustic wave propagating in the viscoelastic film loses a portion of its energy to the liquid. However, if $Z_{1} \ll Z_{0}$, the acoustic wave sees a low impedance boundary at the coating/liquid interface and essentially all of the energy is reflected back into the viscoelastic layer. This latter case is representative of most liquid-phase sensor systems, in which high modulus polymers are used as coatings and low viscosity Newtonian liquids, primarily water, contact the coating. Under these conditions, the total surface impedance is a simple linear combination of the motional impedances due to the liquid and a finite thickness viscoelastic film. It is noted that when the TSM resonator is only loaded with a viscoelastic 
coating, $Z_{1}=0$, and eq (5) reduces to the input impedance at the quartz surface/coating interface: $:^{12}$

$$
Z_{\mathrm{S}}=j Z_{0} \tan \left(\omega\left(\frac{\rho_{f}}{G}\right)^{1 / 2} h_{f}\right)
$$

In obtaining eq (6), it is noted that the identity $\tan (j x)=j \tan (x)$ has been used. $Z_{0}$ is the characteristic impedance of the viscoelastic coating, and is given by $\left(\rho_{f} G\right)^{1 / 2}$.

Polymer films are commonly applied as sorbing layers in gas and liquid-sensing applications. ${ }^{1-4}$ More recently, polymer materials are being investigated as effective wave-guiding layers in the implementation of guided shear-horizontal surface acoustic wave (SH-SAW) sensor platforms. ${ }^{5}$ Polymers are viscoelastic materials and their elastic properties can be described by a complex modulus, $G=G^{\prime}+j G^{\prime \prime}$. It is important to understand how the TSM resonator responds to a polymer coating.

Because the shear modulus $G$ is a complex number, the mechanical impedance described by eq (6) does not readily decompose into analytical expressions for $R_{2}$ and $L_{2}$. Thus, approximate forms of $Z_{\mathrm{s}}$ are needed. The tangent function can be expanded in a Taylor series and the first two terms are retained, $\tan (x) \approx x+1 / 3 x^{3}$. This leads to: ${ }^{10}$

$$
\tan \left(\omega h_{f} \sqrt{\frac{\rho_{f}}{G}}\right) \approx \omega h_{f} \sqrt{\frac{\rho_{f}}{G}}+\frac{1}{3} \omega^{3} h_{f}^{3}\left(\frac{\rho_{f}}{G}\right)^{3 / 2}
$$

The surface mechanical impedance of the viscoelastic layer can then be written as:

$$
Z_{\mathrm{s}} \approx \frac{\omega^{3} \rho_{f}^{2} h_{f}^{3} G^{n}}{3|G|^{2}}+j\left(\omega \rho_{f} h_{f}+\frac{\omega^{3} \rho_{f}^{2} h_{f}^{3} G \prime}{3|G|^{2}}\right)
$$

The first term (the real component of $Z_{\mathrm{S}}$ ), proportional to $G^{\prime \prime}$ the attenuation due to the coating. The second term (the imaginary component of $Z_{\mathrm{s}}$ ) represents the energy storage in the film; it consists of a mass component, $\omega \rho_{f} h_{f}$, and a viscoelastic component proportional to $G^{\prime}$.

The resistance $R_{2}$ and inductance $L_{2}$ due to the viscoelastic layer can be deduced from eqs. (2), (3), and (7) or (8):

$$
\begin{gathered}
R_{2}=\frac{N \pi}{4 K_{0}^{2} \omega_{\mathrm{s}} Z_{\mathrm{q}} C_{0}}\left(\frac{\omega_{s}^{3} \rho_{f}^{2} h_{f}^{3} G{ }^{\prime \prime}}{12}\right) \\
L_{2}=\frac{N \pi}{4 K_{0}^{2} \omega_{\mathrm{s}}^{2} z_{\mathrm{q}} C_{0}}\left(\omega_{\mathrm{s}} \rho_{f} h_{f}+\frac{\omega_{s}^{3} \rho_{f}^{2} h_{f}^{3} G \prime}{3|G|^{2}}\right)
\end{gathered}
$$

By setting 


$$
\begin{gathered}
A=\frac{G \prime}{|G|^{2}}=\frac{12 R_{2} K_{0}^{2} C_{0} Z_{\mathrm{q}}}{N \pi \omega_{\mathrm{s}}^{2} h^{3} \rho_{f}^{2}} \\
B=\frac{G \prime \prime}{|G|^{2}}=\frac{12 K_{0}^{2} \omega_{s} C_{0} Z_{\mathrm{q}} L_{2}-3 \rho_{f} h_{f} N \pi}{N \pi \omega_{\mathrm{s}}^{2} h^{3} \rho_{f}^{2}}
\end{gathered}
$$

both the storage modulus $G^{\prime}$ and loss modulus $G^{\prime \prime}$ can be extracted as

$$
\begin{aligned}
& G^{\prime}=\frac{B}{A^{2}+B^{2}} \\
& G^{\prime \prime}=\frac{A}{A^{2}+B^{2}}
\end{aligned}
$$

A more precise approximation can be obtained by retaining the first three terms of the Taylor series. ${ }^{14}$ In this case,

$$
G^{\prime}=\frac{B}{A^{2}+B^{2}} \text { and } G^{\prime \prime}=\frac{A}{A^{2}+B^{2}}
$$

where

$$
\begin{gathered}
A=\left(\frac{\left(\frac{19 \rho_{f} h}{24}-\frac{L_{2}}{A_{0}}\right)+\left[\left(\frac{L_{2}}{A_{0}}-\frac{19 \rho_{f} h}{24}\right)^{2}+\frac{R_{2}^{2}}{\omega_{\mathrm{S}}^{2} A_{0}^{2}}\right]^{1 / 2}}{\frac{4 \omega_{\mathrm{S}}^{4} \rho_{f}^{3} h^{5}}{15}}\right)^{1 / 2} \\
B=\frac{15 R_{2}}{4 \omega_{\mathrm{S}}^{5} \rho_{f}^{3} h^{5} A_{0}} \frac{1}{A}-\frac{5}{4 \omega_{S}^{2} h^{2} \rho_{f}}
\end{gathered}
$$

with

$$
A_{0}=\frac{N \pi}{4 K_{0}^{2} \omega_{s} C_{0}} \frac{1}{\sqrt{\mu_{\mathrm{q}} \rho_{\mathrm{q}}}}
$$

\section{Experimental}

Polymer layers are spin-coated on the TSM resonators using SU-8-2002 solution, used as received from MicroChem Corp. The films are soft baked by heating on a hot plate at 65 and $95^{\circ} \mathrm{C}$, for $2 \mathrm{~min}$ at each temperature, to remove solv ent and densify the film. Films are then exposed to a 15-W UV lamp for $15 \mathrm{~min}$, followed by a postexposure soft baking sequence identical to the initial soft bake procedure. The UV exposure serves to generate acid that initiates the thermal epoxy crosslinking step. Selected films are also subjected to a hard bake treatment by heating in an oven at $180^{\circ} \mathrm{C}$ for $1 \mathrm{~h}$ to further crosslink the SU-8.

Multiple coatings are used to obtain a range of film thickness up to $22 \mu \mathrm{m}$. Determination of the thickness is very critical for accurate extraction of the shear moduli. A profilometer ( $\alpha$-step $100)$ is used to determine the thickness of each SU-8 film. The measured thickness from the profilometer concurs with the calibration of thickness versus spin-coating conditions as provided by the manufacturer. 
The 9-MHz AT-cut quartz resonators used in this study have polished and lapped planar faces. They are patterned with two concentric electrodes that have a wrap around geometry that allows both electrodes to be electrically excited from one side. The electrodes consist of a 160-nm layer of gold on 15-nm adhesive layer of chromium. The larger electrode (diameter: 12.9 $\mathrm{mm}$ ), used for polymer coating, functioned as the ground electrode. The smaller electrode on the opposing side has a diameter of $6.6 \mathrm{~mm}$. Clean and uncoated TSM resonators are first characterized on the network analyzer to determine the equivalent circuit parameters for the unperturbed device near the resonance. Impedance is measured at the fundamental resonance frequency as well as the third harmonic frequency. $R_{1}, L_{1}$, and $C_{1}$ and $C_{\mathrm{p}}$ can be obtained at this stage by fitting the experimental electrical impedance with the theoretical model of unperturbed TSM device [with $R_{2}=0$ and $L_{2}=0$ in Fig. 1(a)]. These parameters for the unperturbed device are stored for later use. Once thin films are spin-deposited, further measurements are gathered for the reflection coefficients. Reflection coefficients $\left(S_{11}\right)$ are recorded as a function of the frequency near resonance, and are used to determine the complex impedance of the TSM device as:

$$
Z(f)=Z_{0 \mathrm{C}} \frac{1+S_{11}(f)}{1-S_{11}(f)}
$$

where $Z_{0 \mathrm{C}}$ is the characteristic impedance of the measurement system, which is $50 \Omega$ in this case. The resistance $\left(R_{2}\right)$ and inductance $\left(L_{2}\right)$ for the electrical equivalent circuit in Figure $1(\mathrm{~b})$ can be determined from the stored parameters $\left(R_{1}, L_{1}\right.$, and $\left.C_{1}\right)$ for the uncoated device and the reflection coefficient data collected for the coated device as follows:

$$
\begin{aligned}
& R_{\text {total }}=\frac{1}{Y_{\operatorname{maxf}}(f)} \\
& R_{2}=R_{\text {total }}-R_{1} \\
& L_{2}=\frac{1}{\left(\omega_{\mathrm{s}}\right)^{2} C_{1}}-L_{1}
\end{aligned}
$$

where $Y_{\operatorname{maxf}}(f)$ represents the peak value of the admittance as a function of the frequency near resonance.

Experimentally obtained impedance data and film thicknesses measured using the profilometer are then used in the analysis. The density of the SU-8 film $\rho_{f}$ is taken to be 1.2 $\mathrm{g} / \mathrm{cm}^{3}$, as provided by the manufacturer. The analysis is then performed for the extraction of the shear moduli as indicated by eq (9)-(16). Modeling of viscoelastic films involves finding the solutions to five parameters $\left(G^{\prime}, G^{\prime \prime}, h_{f}, \rho_{f}\right.$, and $\left.C_{\mathrm{p}}\right)$. The parasitic capacitance $C_{\mathrm{p}}$ is included 
for the fact that $C_{\mathrm{p}}$ is closely related to the supporting structure, which may change substantially after the coating. However, admittance measurements contribute only two parameters-the real and imaginary part of mechanical impedance $\operatorname{Re}\left(Z_{\mathrm{s}}\right)$ and $\operatorname{Im}\left(Z_{\mathrm{s}}\right)$. Both $h_{f}$ and $\rho_{f}$ are used as constraints in the modeling programs. Usually a tight constraint on $h_{f}$ and $\mathrm{p}_{f}$ can generate unique solutions of $G^{\prime}$ and $G^{\prime \prime}$.

A coated TSM resonator is put in an oven and connected to the Network Analyzer via a SMA connector and coaxial cable for the temperature-dependence studies. A thermal couple is attached in contact with the measurement cell for accurate control of temperature. For the investigation of the polymer film in liquid environments, deionized (DI) water is injected into the measurement cell using a glass pipette. The resonant frequency and the admittance at the resonant frequency are recorded as a function of time after exposure to water.

\section{Results}

Admittance magnitudes for the bare and SU-8-coated 9-MHz TSM quartz resonator are shown in Figure 2. As expected, the resonant frequencies of the coated devices are shifted to lower values and the admittance magnitudes exhibit damping due to the device loss, as the coating thickness increases. Accordingly, the TSM resonator experiences a drop in the quality factor ( $Q$-factor), which can be observed by the widening of the series resonant peak. When the thickness of SU-8 film reaches $5 \mu \mathrm{m}$, the resonant frequency shift apparently begins to deviate from the linear region and approaches its nongravimetric regime, as indicated in Figure 3 . This implies that the viscoelastic properties of the polymer gain influence on the device response and significant deformation occurs on the upper film surface.

The dynamical behavior of the viscoelastic film's displacement across the thickness $h_{f}$ is determined by the acoustic phase shift, $\phi$, across the film. ${ }^{7,12}$ If the film is sufficiently thin and rigid so that $\phi \ll \pi / 2$, then the entire film tends to move synchronously with the resonator surface and no strain occurs in the film. The polymer film deformation is negligible only when the thickness of the film is much thinner than the acoustic wavelength. Beyond that range, the relation between the frequency shift and the mass loading is nonlinear because the acoustic delay in the overlay is no longer negligible.

In the case of the SU-8-coated device, the phase velocity across the polymer film can be estimated as follows:

$$
v=\left(\frac{G}{\rho}\right)^{1 / 2} \approx\left(\frac{1.6 \times 10^{10} \mathrm{dyne} / \mathrm{cm}^{2}}{1.2 \mathrm{~g} / \mathrm{cm}^{3}}\right)^{1 / 2} \approx 1.16 \times 10^{5} \mathrm{~cm} / \mathrm{s}
$$


Using the value of shear modulus, $G=1.6 \times 10^{10} \mathrm{dyne} / \mathrm{cm}^{2}$ from our experimental results and the film density $\rho=1.2 \mathrm{~g} / \mathrm{cm}^{3}$, the phase shift $\phi$ across the layer of $5-\mu \mathrm{m}$ polymer film is:

$$
\phi=2 \pi f \times h / v \approx 0.155 \times \frac{\pi}{2}<\frac{\pi}{2}
$$

Therefore, a linear relationship between frequency shift and film thickness is observed in the range of about $0-5 \mu \mathrm{m}$. Beyond that, frequency shift increases more rapidly with phase shift $\phi$ (in other words, the thickness of the film) until $\phi=2 / \pi$. In this region, one can determine the shear modulus of the films and study the crosslinking of polymer films initiated by heat or light exposure.

The above discussion is confirmed by the measured device resistance $R\left(R=R_{1}+R_{2}\right)$ shown in Figure 4(a) for a device coated with SU-8 films at varying thicknesses. The resistance $R$ represents the mechanical energy dissipation of the system. $R$ remains very low (less than $20 \mathrm{ohm}$ ) when $0<h_{f}<5 \mu \mathrm{m}$ [corresponding to a phase shift $\phi<0.155 \times(\pi / 2)$ ]. The damping of the admittance spectrum is not really significant in this region as indicated in Figure 2. For film thickness such that $5 \mu \mathrm{m}<h_{f}<30 \mu \mathrm{m}, R$ increases sharply first and gradually approaches a maximum value. Film resonance occurs when $\phi=\pi / 2$, which corresponds approximately to $h_{f} \cong 30 \mu \mathrm{m}$. Film resonance represents the strongest coupling between the resonator and the coated film, resulting in a greater extraction of acoustic energy from the resonator. $R$ reaches its maximum at film resonance represents the strongest coupling between the resonator and the coated film, resulting in a greater extraction of acoustic energy from the resonator. $R$ reaches its maximum at film resonance. ${ }^{12}$

The resistance change as a function of the film thickness in Figure 4(a) concurs with the results reported by Martin and Frye ${ }^{7}$ for another polymer film. The resistance is approaching but has not reached the maximum value at the thickness of $22 \mu \mathrm{m}$. This result is quite consistent with our prediction that the film resonance occurs at around $h_{f} \cong 30 \mu \mathrm{m}$. Furthermore, it verifies the reliability of our fitting results of the shear modulus $(G)$ for the SU-8 film.

The Sauerbrey equation indicates that the frequency shift is proportional to the square of the fundamental frequency of the TSM resonator. Therefore, the sensitivity/resolution of the device may be improved by operating the TSM resonator at higher odd harmonics. The use of multiple odd harmonics also allows one to solve the problem of uniqueness of fit for the viscoelastic films on TSM resonator. When the film density and thickness are not available, a unique solution will not be obtained if the crystal impedance measurements are made only at the 
fundamental frequency. By measuring the electrical response of the TSM resonator at various harmonics, more input variables are introduced.

Measurements were also performed with the TSM resonator operating at the third harmonic. The phase shift of third harmonic across the polymer coating on TSM resonator is three times that of the fundamental frequency, which means that a larger "effective" thickness is achieved by using the third harmonics. Figure 4(b) shows the loss of the TSM resonator with respect to the film thickness measured at the third harmonic. Both the frequency shift and the resistance of the third harmonic for a film of thickness $h$ are very close to that of the fundamental frequency for a film of a thickness $3 \mathrm{~h}$. In Figure 4(b), the device resistance approaches saturation at a thickness of approximately $10 \mu \mathrm{m}$, agreeing with the prediction that the film resonance occurs at thickness around $10 \mu \mathrm{m}$ for the third harmonic.

Measured series-resonant frequency shifts, relative to the uncoated device, versus polymer thickness are also shown in Figure 5(a) for both hard-baked and soft-baked coatings. Both sets of data exhibit a continuous decrease in frequency with increasing thickness and the shifts for the hard-baked coatings are lower in magnitude than for the soft-baked coatings. One factor that may contribute to the observed difference in frequency shifts is that thermal processing lowers the coating mass via desorption of the solvent. ${ }^{11,12}$ SU-8 2000 photoresists use a mixed solvent consisting of $1-5 \%$ propylene carbonate (boiling point: $240{ }^{\circ}$ ) and $23-78 \%$ cyclopentanone (boiling point: $130-131^{\circ} \mathrm{C}$ ). The low t emperature and short thermal processing duration of soft-baked films may be insufficient to remove all solvent residue inside the polymer. In addition, the residual solvent inside the soft-baked polymer film may play a role as plasticizer. ${ }^{13}$ The plasticizing effect by the solvent results in a rise of the loss modulus $\left(G^{\prime \prime}\right)$. Therefore, uncured device suffers a higher loss than the cured one as indicated in Figure 5(b). In each case, the device resistance $R$ increases with increasing layer thickness, and the values are higher for the uncured coatings, as expected due to the reason above. In addition, the hard-baked film is expected to have a higher degree of crosslinking, which would also contribute to the observed differences in frequency shift and shear modulus.

Figure 6 shows the results for shear moduli extracted from measurements made at both the fundamental frequency and third harmonic for the SU-8-coated TSM device. The moduli increase with film thickness and the shear storage modulus exceeds the shear loss modulus by more than an order of magnitude. As the film thickness increases above $10 \mu \mathrm{m}$, both the storage modulus $\left(G^{\prime}\right)$ and loss modulus $\left(G^{\prime \prime}\right)$ approach saturation, indicating that the viscoelastic properties are now independent of thickness. A thicker film layer is desirable for this analysis because of the very small viscoelastic contribution resulting from a thin film layer. For a relatively 
small film layer thickness, the extracted viscoelastic properties do not reflect that of a bulk film material. This essentially means that the phase shift across the thin film layer is practically negligible. Under these conditions, the extraction of the storage and loss modulus could be inaccurate. These figures confirm earlier discussion that meaningful values of viscoelastic properties can only be obtained at sufficiently large polymer thicknesses. The storage modulus $\left(G^{\prime}\right)$ of the SU-8 film (assuming $\rho=1.2 \mathrm{~g} / \mathrm{cm}^{3}$ ) approaches its saturation value of $1.66 \times$ $10^{10}$ dyne $/ \mathrm{cm}^{2}$ for relatively thicker films $(10-20 \mu \mathrm{m})$, while the loss modulus $\left(G^{\prime \prime}\right)$ reaches its maximum at $6.0 \times 10^{8} \mathrm{dyne} / \mathrm{cm}^{2}$ in the same range of thickness. The accuracy of the extracted shear modulus parameters occurs at high thickness where viscoelastic contribution is substantial. It is noted that, at relatively high thickness, the measured loss approaches saturation as shown in Figure 4. The modeling used here will result in large errors for acoustically thin films and for rigid layers, because the loss contribution due to the coating can be very small (coating mostly represents a mass load), and because the extraction depends on the measured loss.

For relatively small film layer thicknesses, the electrical response of the devices in this "gravimetric" region is only dependent upon the surface mass density $\rho_{\mathrm{S}}$, and not upon $G^{\prime}$ and $G^{\prime \prime}$. These figures confirm earlier discussion that meaningful values of viscoelastic properties can only be obtained at sufficiently large polymer thickness. It must be pointed out that the polymer thickness and density are the major sources of error in the extractions.

Apparently, the polymer film is in a state close to a glassy state due to the high value of $G^{\prime}$ and high ratio of $G^{\prime}$ to $G^{\prime \prime}$. The storage modulus of SU-8 is comparable to that of PMMA, which was reported to be $1.43 \times 10^{10}$ dyne $/ \mathrm{cm}^{2}$ at a thickness of $17.6 \mu \mathrm{m} .^{14}$

Operating the TSM resonator at higher odd harmonic offers some advantages over using only the fundamental frequency. The TSM device sensitivity/resolution may be improved by using higher frequency. The use of higher harmonics also allows one to solve the problem of uniqueness of fitting for the viscoelastic film, which has an unknown density and/or thickness. The fitting program involves converting electrical response (the real and imaginary part of film electrical impedance) to film properties $\left(h_{f}, \rho_{f}, G^{\prime}\right.$, and $\left.G^{\prime \prime}\right)$. When the film density and thickness are not available, a unique solution will not be obtained if the crystal impedance measurements are made only in the fundamental frequency. By measuring the electrical response of the TSM resonator at various harmonics, more input variables are introduced. ${ }^{10,15}$ However, this method overlooks the frequency dependence of shear moduli in the viscoelastic regime. This method has only been successfully applied for uniform films of linear mono-disperse polymer. ${ }^{16}$

Figure 6 also shows the results of the storage and loss moduli extracted from the third 
harmonic frequency data for SU-8 film. Both the storage modulus and loss modulus become essentially independent of film thickness for measurements made at the third harmonic sooner than for measurements made at the fundamental frequency. This is another advantage of using third harmonic over using fundamental frequency, because bulk shear modulus value can be obtained by using a thinner film. One means of interpreting the moduli for the higher harmonic frequencies is that a larger "effective" thickness of material is achieved. In the 9- to 27-MHz range, very little frequency dependence of the loss modulus is observed. However, the storage modulus measured at the third harmonic is higher than that measured at the fundamental frequency. The difference observed with the fundamental and third harmonic response may be attributed to the differences in crystal motion and vibration amplitude at the different harmonics.

Temperature is a significant environmental parameter that affects the performance of a TSM resonator. In addition, the viscoelastic properties of polymer film show strong dependences on the temperature and operating frequency of the measurements. The temperature dependence of the SU-8-coated device, obtained at the fundamental frequency, is determined for a $10 \mu \mathrm{m}$ SU-8 coating. Figure 7(a) shows that the film resistance $\left(R_{2}\right)$ increases as a function of temperature. $R_{2}$ is less dependent on the temperature at very low temperature. When the temperature is lower than $-60^{\circ} \mathrm{C}$, the change of the film resistance shows the smallest rate of change; one can assume the polymer film to be even more glassy than at room temperature. It is noted that, because the bare crystal exhibits no change in response over this temperature range, the effects observed here are mainly due to changes of the viscoelastic properties of the film. Figure 7 (b) shows the temperature dependence of the storage modulus. Similarly, a rapid decrease of the slope of $G^{\prime}$ with temperature was observed at low temperature.

Values of $G^{\prime}$ were first determined assuming no change in the height or the density of the SU-8 film over the $115^{\circ} \mathrm{C}$ temperature range and are shown in Figure $7(\mathrm{~b})$ as diamonds connected with a dashed line. Thermal expansion coefficients (TEC) for SU-8 have recently been reported. ${ }^{17}$ The volumetric TEC, $\alpha_{\mathrm{v}}$, was found to be $457 \mathrm{ppm}{ }^{\circ} \mathrm{C}^{-1}$ and the out-of-plane TEC, $\alpha_{2}$, was determined to be $253 \mathrm{ppm}^{\circ} \mathrm{C}^{-1}$. Using the reported TEC values, new film thicknesses and densities were calculated for each temperature, assuming a thickness of $10 \mu \mathrm{m}$ and a density of $1.2 \mathrm{~g} \mathrm{~cm}^{-3}$ for the film at $20^{\circ} \mathrm{C} . G^{\prime}$ values were extracted using the corrected film parameters and the adjusted values are shown in Figure $7(\mathrm{~b})$ as triangles connected with a solid line. At $-75^{\circ} \mathrm{C}$, using the adjusted values of fi Im height and density, $9.76 \mu \mathrm{m}$ and $1.25 \mathrm{~g}$ $\mathrm{cm}^{-3}$, respectively, results in extracted values of $G^{\prime}=1.15 \times 10^{10}$ dyne $\mathrm{cm}^{2}$ and $G^{\prime}=1.10 \times$ $10^{8}$ dyne $\mathrm{cm}^{2}$. The adjusted values are slightly larger than the corresponding values obtained assuming no temperature effect on the film, $G^{\prime}=1.01 \times 10^{10}$ dyne $\mathrm{cm}^{2}$ and $G^{\prime \prime}=8.38 \times$ 
$10^{7}$ dyne $\mathrm{cm}^{2}$. Similarly, at $40{ }^{\circ} \mathrm{C}$ the adjusted height of $10.05 \mu \mathrm{m}$ and density of $1.189 \mathrm{~g} \mathrm{~cm}^{3}$ results in $G^{\prime}=7.95 \times 10^{9}$ dyne $\mathrm{cm}^{2}$ and $G^{\prime \prime}=3.49 \times 10^{8}$ dyne $\mathrm{cm}^{2}$, slightly smaller than the values obtained without the temperature correction, $G^{\prime}=8.12 \times 10^{9}$ dyne $\mathrm{cm}^{2}$ and $G^{\prime \prime}=3.66 \times$ $10^{8}$ dyne $\mathrm{cm}^{2}$ dyne $\mathrm{cm}^{2}$. Although film thickness and density do affect the extracted shear moduli, clearly the general trend in temperature-dependent behavior can primarily be attributed to changes in the polymer viscoelastic behavior.

A primary requirement for use of a polymer as a wave-guiding layer for a guided SH-SAW sensor platform in aqueous environments is the stability of the polymer in water. ${ }^{5,18}$ The frequency response of a device operated in a liquid environment reflects the combined effects of changes in viscoelastic properties and mass loading effect of surface film with the mechanical properties of the liquid overlayer. In this study, the SU-8-coated TSM device was exposed to DI water to study the stability of the polymer in water.

Figures 8(a) and 8(b) show the frequency and device resistance after the addition of the water to the surface of TSM device coated with a 10- $\mu \mathrm{m}$ SU-8 film. More data points are needed in the first $10 \mathrm{~min}$ due to the rapid change during this time range. The exposure to DI water results in a considerable damping of the admittance, with the resistance of the coated devices rising from $105 \mathrm{ohm}$ to a $191 \mathrm{ohm}$ within $5 \mathrm{~s}$, while at the same time, the resonant frequency of the device shifts by $1.7 \mathrm{kHz}$, which is almost identical to the frequency change of an uncoated device exposed to water. Therefore, the sharp change in the first $5 \mathrm{~s}$ should be mainly attributed to the addition of an extra semiinfinite Newtonian liquid overlayer on top of the polymer layer.

The subsequent change in loss and frequency shift is mainly due to sorption process (either adsorption onto the surface or absorption into the three-dimensional matrix). Both the resistance and frequency shift increase with water uptake, but with a diminishing slope before the sorption attains equilibrium around $t=400 \mathrm{~s}$. It is interesting to note that the sorption of water molecules causes a small change in the resistance of the device, with resistance rising from 191 ohm to 195 ohm. Meanwhile, a significant frequency shift was observed, changing from 1.7 to $4.1 \mathrm{kHz}$. The observed frequency change, in the limit of mass-loading only, where the Sauerbrey equation is valid, corresponds to an added mass of approximately $2.1 \mu \mathrm{g}$.

There are two limiting case models for polymer sorption kinetics. ${ }^{19}$ Case I behavior, where the sorption process exhibits Fickian-diffusion kinetics, is characterized as having a square-root-of time dependence of the sorption curve. Case II behavior exhibits a linear time dependence, because the diffusion is fast compared to the relaxation of polymer and the later becomes the rate-limiting process. Figure $8(\mathrm{~b})$ shows that Case I behavior is followed at short times since the plot of $\Delta f$ versus $t^{1 / 2}$ is linear over a substantial portion of the sorption curve [refer 
to the inset of Fig. 8(b)]. Assuming that observed frequency shifts in the time range of 5-150 s can be attributed primarily to mass-loading effects, then the ratio of mass sorbed into the SU-8 coating at time $\mathrm{t}$ to the sorbed mass at time $t=$ infinity $\left(M_{\mathrm{t}} / M_{\mathrm{t}}=\infty\right)$ is equal the ratio of frequency shifts at the same times. The short-time limit for Fickian diffusion can be used to obtain an effective value for the diffusion coefficient, $D$, using the following equation: ${ }^{19}$

$$
\frac{M_{\mathrm{t}}}{M_{\mathrm{t}=\infty}}=\frac{\Delta f_{\mathrm{t}}}{\Delta f_{\mathrm{t}=\infty}}=\frac{2}{h}\left[\frac{D t}{\pi}\right]^{1 / 2}
$$

where $h$ is the film thickness. Fitting the data shown in Figure 7(b) to this model results in $D=1.25( \pm 0.14) \times 10^{-9} \mathrm{~cm}^{2} \mathrm{~s}^{-1}$.

The diffusion coefficient estimated here for SU-8 is similar to values reported recently for other photoresists $\left(0.28-3.510^{-9} \mathrm{~cm}^{2} \mathrm{~s}^{-1}\right)^{20}$ and is approximately an order of magnitude lower than range $\left(1-1.99 \times 10^{-8} \mathrm{~cm}^{2} \mathrm{~s}^{-1}\right)$ given ${ }^{21}$ for another commonly used wave-guiding material for SH-SAW applications, poly(methyl methacrylate) (PMMA). This suggests that SU-8 will be relatively stable with respect to water uptake. However, it should be noted that based on the shear moduli determined here, that SU-8 appears to be in a nearly glassy state where non-Fickian (or anomalous) diffusion kinetics might be observed, ${ }^{22}$ suggesting that further experiments would be needed to confirm the measured diffusion coefficient, because diffusion and polymer relaxation may be occurring on similar time scales.

In addition, sorption of small molecules from liquid to a polymer film may modify the viscoelastic properties of polymer. ${ }^{14}$ The absorbed solute molecules may behave as a lubricant so that the polymer chains can move more freely and the polymer softens. The magnitude of this effect depends on two factors: the polymer/liquid interaction and the amount of the solute absorbed. The shear moduli of SU-8 film in water were extracted based on a nonlinear multiple-loading model. Because the SU-8 film is relatively thick and cannot be treated as an ideal mass layer, eq (4) must be used for the calculation of the total impedance of the surface overlayers. Fitting the equilibrated (i.e., long-time) data to this model results in $G^{\prime}$ changing from $3.8 \times 10^{9}$ dyne $/ \mathrm{cm}^{2}$ to $1.75 \times 10^{9}$ dyne $/ \mathrm{cm}^{2}$ and $G^{\prime \prime}$ changing from $3.8 \times 10^{7} \mathrm{dyne} / \mathrm{cm}^{2}$ to $1.1 \times 10^{8}$ dyne $/ \mathrm{cm}^{2}$ for when water is added to a $10-\mu \mathrm{m}$ SU-8 film. The thickness and density of the film are assumed unchanged upon exposure to water due to the fact that SU-8 is a rigid film with high degree of crosslinking. It is assumed that the coating swelling is negligible due to the small water uptake. Initial work on the use of SU-8 as a stable wave-guiding layer for guided SH-SAW sensor platforms in liquid environments has confirmed the above assumption, because little or no frequency shift or loss was observed on the coated reference device of the sensor. The accuracy of these extracted values needs to be verified by other methods. 


\section{Conclusions}

The storage moduli $\left(G^{\prime}\right.$ and $\left.G^{\prime \prime}\right)$ of the polymer SU-8 film were extracted using a TSM quartz resonator based on the equivalent circuit of the modified BVD model. The resistance, $R$, of the coated device was measured as a function of the film thickness. $R$ is less than $20 \Omega$ when the thickness of film is less than $5 \mu \mathrm{m}$. A linear relationship between frequency shift and film thickness is observed in the range of about $0-5 \mu \mathrm{m}$, beyond which viscoelastic properties significantly contribute to the frequency response. Hence, the accuracy of the extracted shear modulus parameters occurs at relatively high thickness where viscoelastic contribution is substantial. However, the bulk shear modulus value can be reached at a smaller thickness by using the third harmonic instead of the fundamental frequency. Apparently, the polymer film is in a near glassy state due to the high value of $G^{\prime}$ and high ratio of $G^{\prime}$ to $G^{\prime \prime}$. The storage modulus of SU-8 is found to be comparable to that of PMMA, which was reported to be $14.3 \times 10^{10}$ dyne/ $\mathrm{cm}^{2}$ at a thickness of $17.6 \mu \mathrm{m}$. SU-8 shows a continuous decrease of the storage modulus with increasing temperature. However, below $-60{ }^{\circ}$, the ra tio of $G^{\prime}$ to $G^{\prime \prime}$ becomes even higher than at room temperature, indicating a glassy state.

Film resonance is observed when the SU-8 film thickness reaches about $30 \mu \mathrm{m}$ (corresponding to a phase shift of $\pi / 2$ ). This result agrees very well with the expected thickness for film resonance, which is calculated from the phase velocity based on our experiment value of the shear moduli of the SU-8 film.

Exposure to water indicates some degree of changes in both $G^{\prime}$ and $G^{\prime \prime}$. However, stability is rapidly reached, indicating relatively lower water uptake than cured PMMA. The Fickian-diffusion model can be used to describe the sorption behaviors of the SU-8 film for water, with an effective value of $1.52 \times 10^{-9} \mathrm{~cm}^{2} \mathrm{~s}^{-1}$ for the diffusion constant at room temperature. These results, combined with the actual extracted values of $G^{\prime}$ and $G^{\prime \prime}$, indicate that SU-8 film can be used as an effective, stable wave-guiding layer in the implementation of guided SH-SAW sensors in liquid environments. It is recalled that the complex shear moduli are utilized to estimate the polymer influence on the velocity shift and attenuation change, hence, on the sensor characteristics. It is noted that most polymers can be used as the thin guiding layer for guided SH-SAW sensors because of their relatively low shear wave velocity and their ease of surface layer preparation. However, their overall acoustic loss, stability in water, and subsequent changes in liquids dictate their selection. 


\section{Acknowledgements}

This work is partially supported by NSF Grant Nos: ECS-9876366, ECS-0110381, and CHE-0074962.

\section{References and Notes}

1. Sauerbrey, G. Zeitschrift Phys 1959, 155, 206-222.

2. Benes, E. J Appl Phys 1984, 56, 608-626.

3. Lee, Y.; Shana, Z. A.; Josse, F. Proceedings of the IEEE Ultrasonics Symposium, IEEE; 94; pp 633-638.

4. Martin, S. J.; Frye, G. C.; Wessendorf, K. Sensors Actuators A 1994, 44, 209-218.

5. Josse, F.; Bender, F.; Cernosek, R. W. Anal Chem 2001, 73, 5937-5944.

6. Martin, S. J.; Granstaff, V. E.; Frye, G. C. Anal Chem 1991, 63, 2272-2281.

7. Martin, S. J.; Frye, G. C. Proceedings of the IEEE Ultrasonics Symposium, IEEE; 1991; pp 393-398.

8. Martin, S. J.; Frye, G. C.; Ricco, A. J.; Senturia, S. D. Anal Chem 1993, 65, 2910-2922.

9. Bandey, H. L.; Martin, S. J.; Cernosek, R. W. Anal Chem 1999, 71, 2205-2214.

10. Wolff, O.; Seydel, E.; Johannsmann, D. Faraday Discuss Chem Soc 1997, 107, 91-104.

11. Shaw, J. M.; Gelorme, J. D.; LaBianca, N. C.; Con-ley, W. E. IBM J R\&D 1997, 41, 95-104.

12. Ballantine, D. S.; White, R. M.; Martin, S. J.; Ricco, A. J.; Zellers, E. T.; Frye, G. C.; Wohltjen, H. Acoustic Wave Sensors: Theory, Design, and Physico-Chemical Applications; Academic Press: San Diego, 1997.

13. Groetsch, J. A.; Dessy, R. E. J Appl Polym Sci 1983, 28, 161-178.

14. Morray, B.; Li, Z.; Hossenlopp, J.; Cernosek, R. W.; Josse, F. Proceedings of the IEEE Frequency Control Symposium and PDA Exhibition, IEEE; New Orleans, LA , 2002; pp 294-300.

15. Domack, A.; Prucker, O.; Ruhe, J.; Johannsmann, D. Phys Rev E 1997, 56, 680 -689.

16. Williams, M. L.; Landel, R. F.; Ferry, J. D. J Am Chem Soc 1955, 77, 3701.

17. Feng, R.; Farris, R. J. J Michromech Microeng 2003, 13, 80 -88.

18. Patel, R. M.; Zhou, R.; Zinszer, K.; Josse, F. Anal Chem 2002, 72, 4888 -4898.

19. Crank, J.; Park, G. S. Diffusion in Polymers; Academic Press: New York, 1968.

20. Berger, C. M.; Henderson, C. L. Polymer 2003, 44, 2101-2108.

21. Linossier, I.; Gaillard, F.; Romand, M.; Feller, J. F. J Appl Polymer Sci 1997, 66, 24665-2473. 
22. Crank, J. The Mathematics of Diffusion; Clarendon Press: Oxford, 1975; 2nd ed., pp 254-255. 
Figure 1

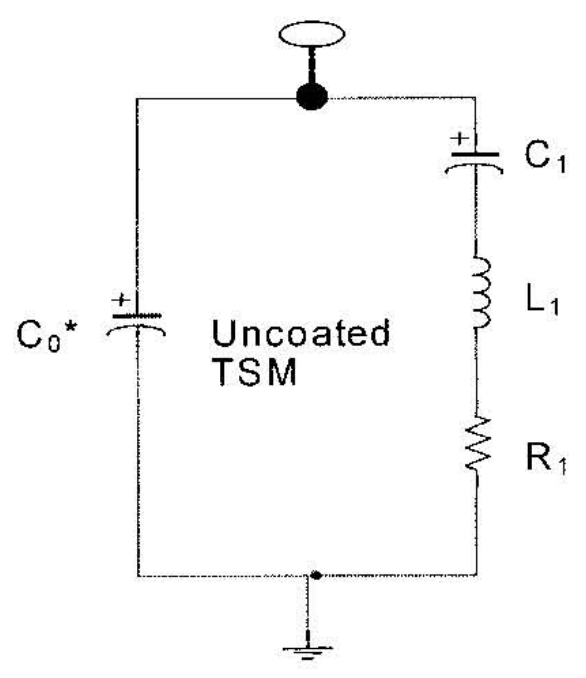

(a)

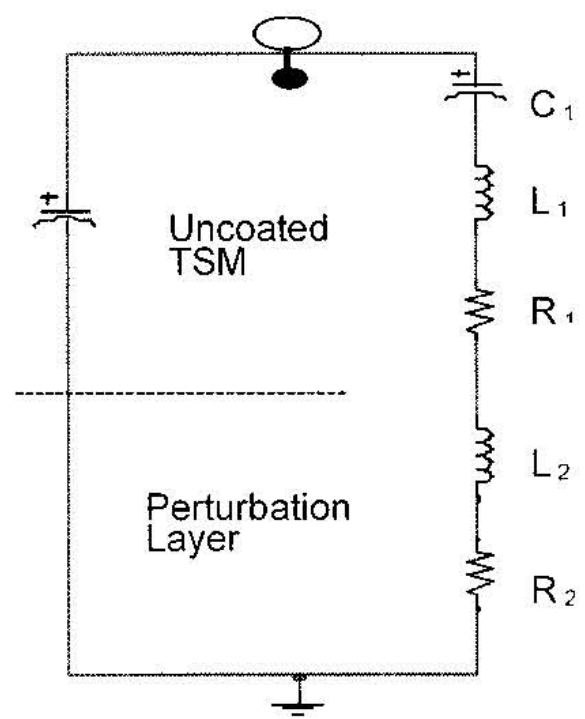

(b)

(a) The BVD model of the unperturbed TSM resonator.

(b) The modified BVD equivalent circuit of the perturbed TSM resonator. 
Figure 2

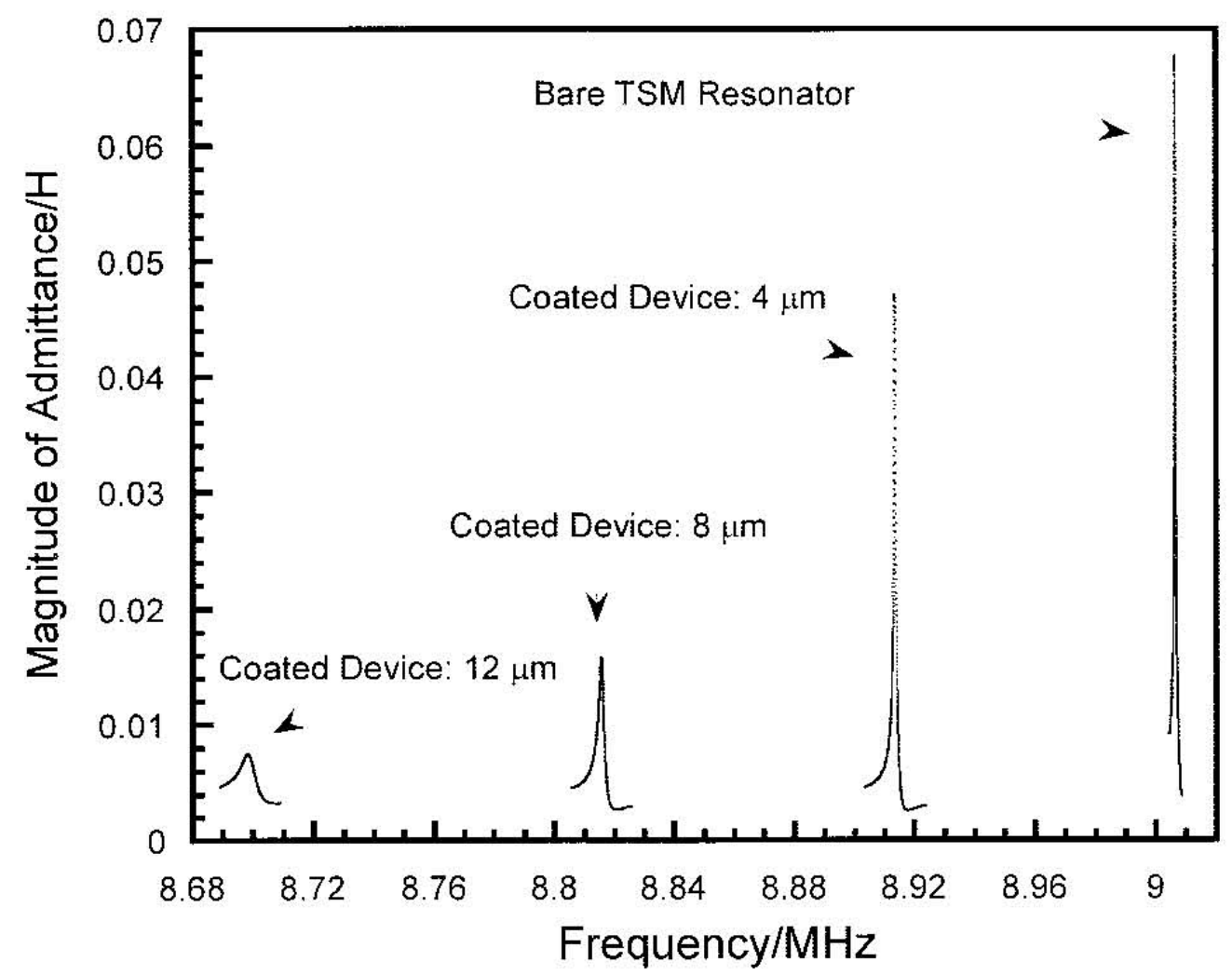

The crystal admittance spectra of the bare and SU-8-coated TSM resonators. 
Figure 3

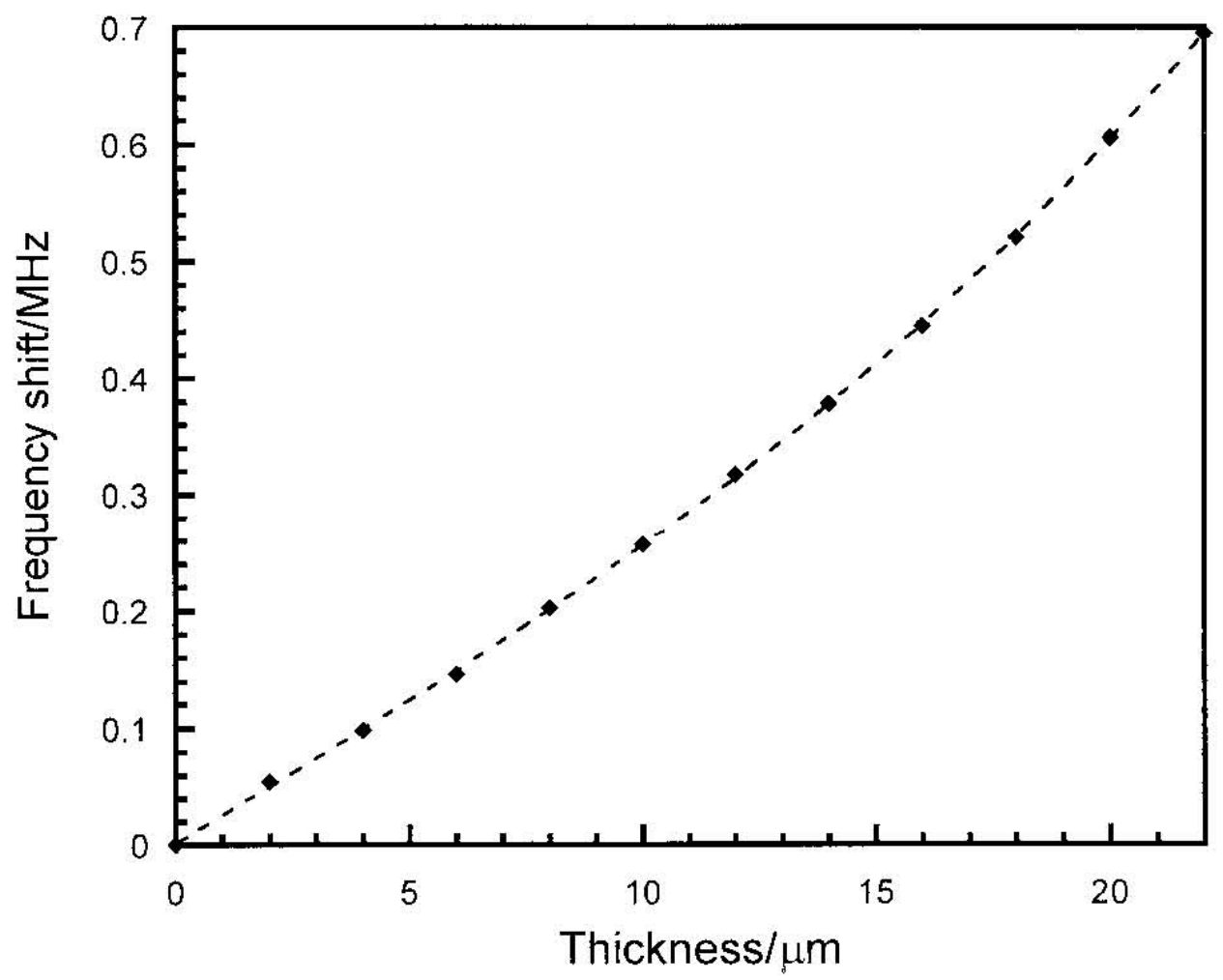

Measured series-resonant frequency shifts as a function of the thickness of SU-8 film in the range of $0-22 \mu \mathrm{m}$ at the fundamental frequency. The device is $9-\mathrm{MHz}$ TSM quartz resonator. 
Figure 4
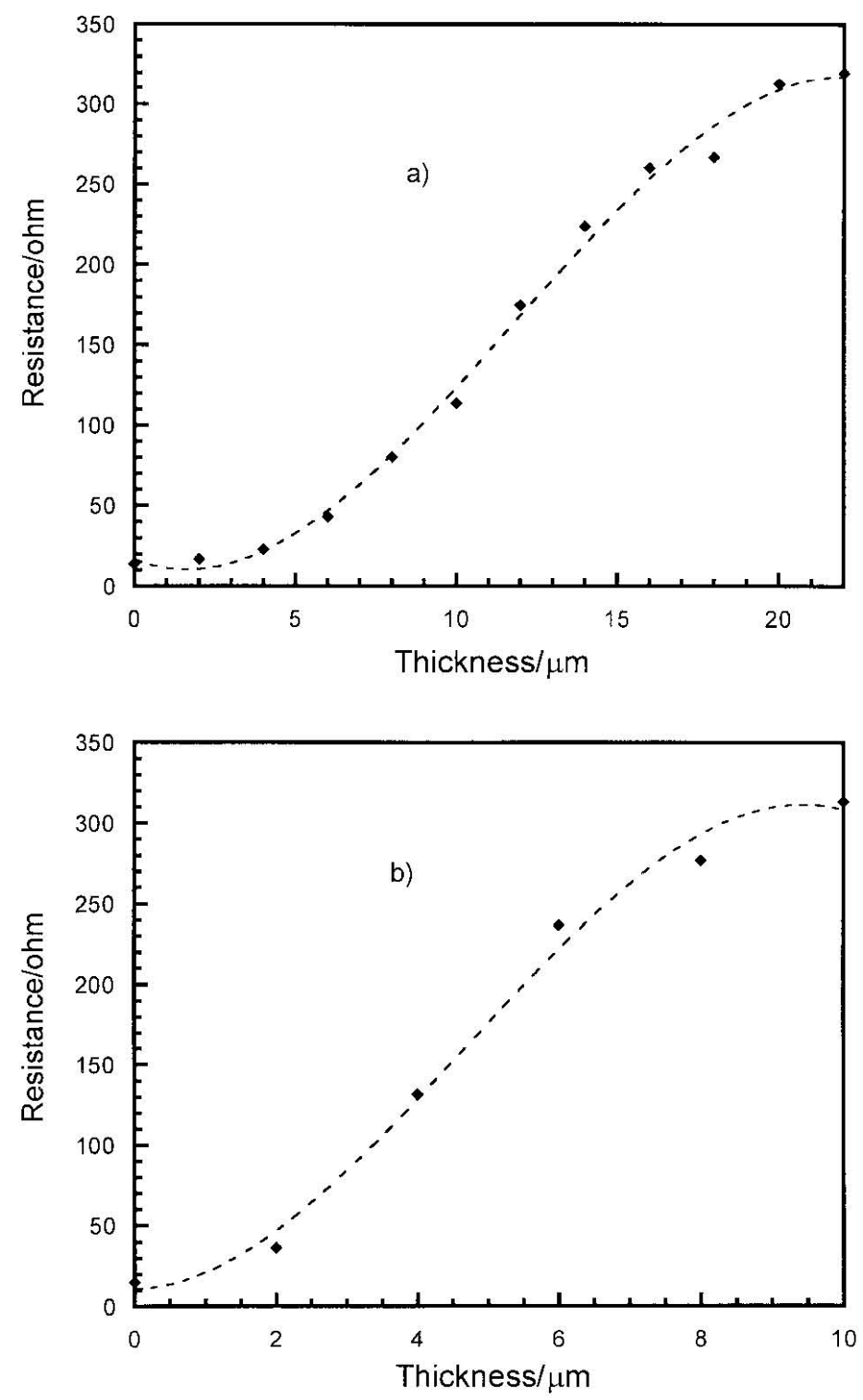

The resistance $R$ of the device $\left(R=R_{1}+R_{2}\right)$ as a function of polymer thickness measured at (a) the fundamental frequency and (b) the third harmonic. The device is $9-\mathrm{MHz}$ TSM quartz resonator. 
Figure 5
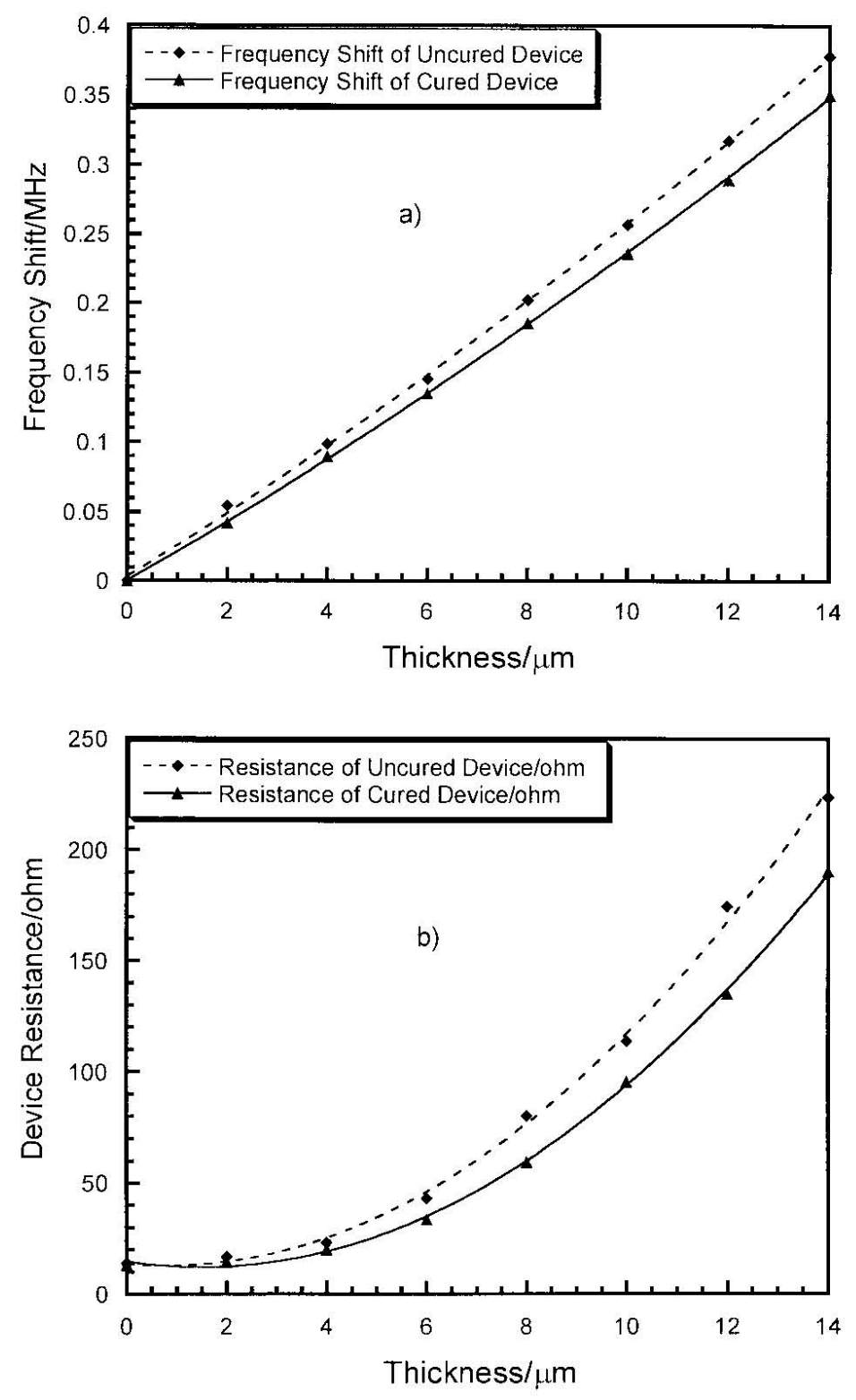

(a) Measured series-resonant frequency shifts.

(b) The resistance $R$ of the device $\left(R=R_{1}+R_{2}\right)$ as a function of polymer thickness for hard-baked (cured) and soft-baked (uncured) coatings. Thicknesses were obtained incrementally. 
Figure 6

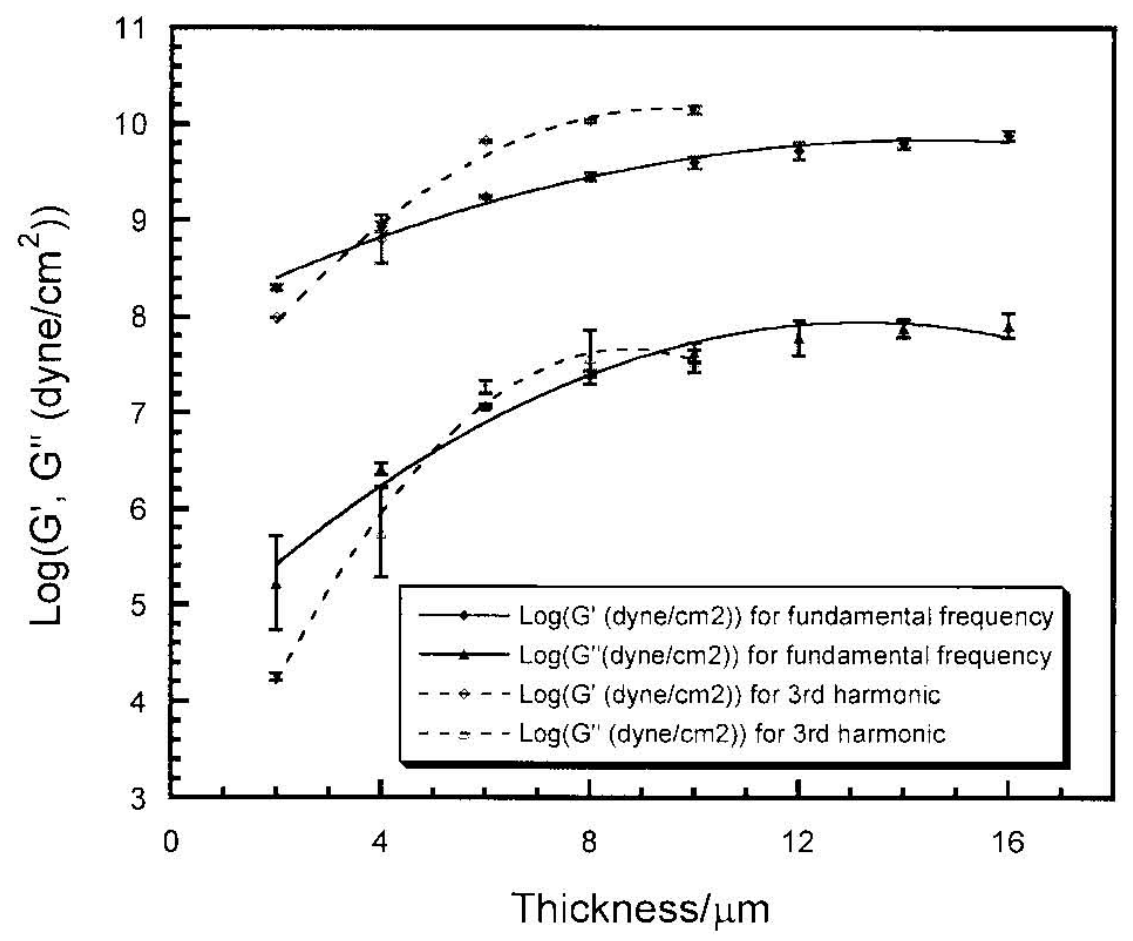

The extracted shear moduli as a function of polymer thickness for SU-8 films using a 9-MHz TSM resonator. Measurements were conducted at the fundamental frequency and third harmonic.

Shown are both storage and loss moduli. The storage moduli of SU-8 film at higher thickness are comparable to those of PMMA. 
Figure 7
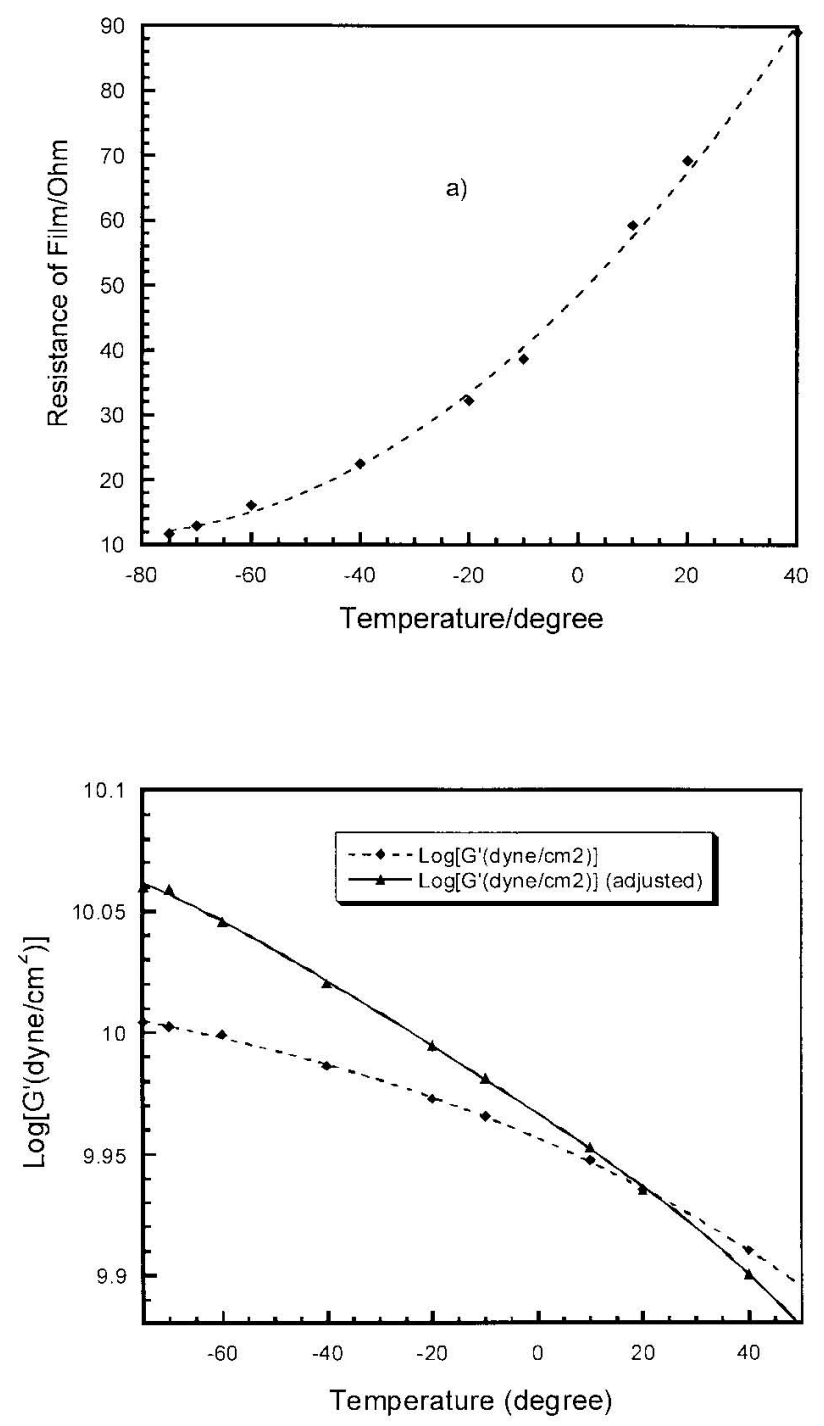

(a) The resistance due to SU-8 coating $\left(R_{2}\right)$ as a function of temperature for a SU-8-coated device at a thickness of $10 \mu \mathrm{m}$.

(b) SU-8 storage modulus $\left(G^{\prime}\right)$ as a function of temperature for the same coating in (a). Adjusted values were determined using film heights and densities corrected for thermal expansion. 
Figure 8
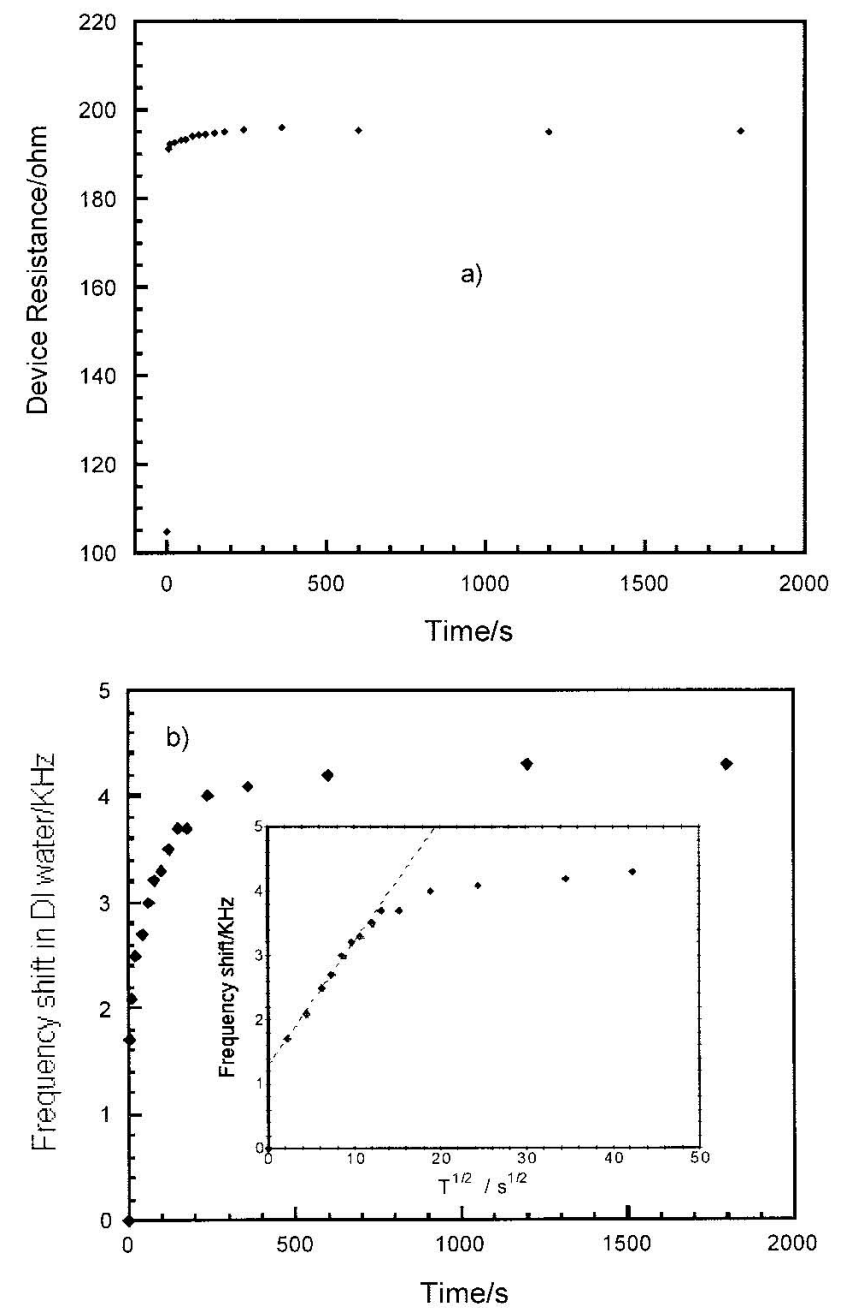

(a) The resistance $R$ of the device $\left(R=R_{1}+R_{2}\right)$.

(b) Measured series-resonant frequency shifts as a function of time for a 10- $\mu \mathrm{m} \mathrm{SU-8-coated}$ device exposed to water. Inset in (b) was the frequency response plotted against $t^{1 / 2}$. The Fickian diffusion model is met in this case. 